PROCEEDINGS OF THE

AMERICAN MATHEMATICAL SOCIETY

Volume 131, Number 11, Pages 3607-3616

S 0002-9939(03)06660-7

Article electronically published on February 24, 2003

\title{
TYCHONOFF EXPANSIONS BY INDEPENDENT FAMILIES
}

\author{
WANJUN HU
}

(Communicated by Alan Dow)

\begin{abstract}
A method for Tychonoff expansions using independent families is introduced. Using this method we prove that every countable Tychonoff space which admits a partition into infinitely many open-hereditarily irresolvable dense subspaces has a Tychonoff expansion that is $\omega$-resolvable but not strongly extraresolvable. We also show that, under Luzin's Hypothesis $\left(2^{\omega_{1}}=2^{\omega}\right)$, there exists an $\omega$-resolvable Tychonoff space of size $\omega_{1}$ which is not maximally resolvable.
\end{abstract}

\section{INTRODUCTION}

A topological space $X$ is called crowded (or dense in itself) if $X$ has no isolated points. E. Hewitt defined a resolvable space as a space which has two disjoint dense subsets (see [17]). A space is called $\kappa$-resolvable [3] if it admits $\kappa$-many pairwise disjoint dense sets. The cardinal $\Delta(X):=\min \{|U|: U$ is a non-empty open subset of $X\}$ is called the dispersion character of the space $X$. The space $X$ is called maximally resolvable [2] if it is $\Delta(X)$-resolvable; if every crowded subspace of $X$ is not resolvable, then $X$ is called hereditarily irresolvable (HI). Analogously, $X$ is open-hereditarily irresolvable (or OHI) if every non-empty open subset of $X$ is irresolvable. V.I. Malykhin introduced a similar concept of extraresolvable (see [20] and [6]): a space is extraresolvable if there exists a family $\left\{D_{\alpha}: \alpha<\Delta(X)^{+}\right\}$of dense susbets of $X$ such that $D_{\alpha} \cap D_{\beta}$ is nowhere dense in $X$ for distinct $\alpha$ and $\beta$. Comfort and García introduced in [6] a similar concept of "strongly extraresolvable" calling a space $X$ strongly extraresolvable if there is a family $\mathcal{D}$ of dense subsets of $X$ such that $|\mathcal{D}|=\Delta(X)^{+}$and $|D \cap E|<n w d(X, \mathcal{T})$ for distinct $D$ and $E$. Here $n w d(X)=\min \{|A|: A \subseteq X$ and $A$ is not nowhere dense in $X\}$. For a survey of recent developments in this area, we refer the reader to [5].

We shall study the following two questions.

Question 0.1. Is every countable $\omega$-resolvable Tychonoff space necessarily strongly extraresolvable?

Question 0.2. Is there an $\omega$-resolvable Tychonoff space which is not maximally resolvable?

Received by the editors October 5, 2001 and, in revised form, June 3, 2002.

2000 Mathematics Subject Classification. Primary 54A25, 05D05; Secondary 54B99.

Key words and phrases. Independent family, KID-expansion, Luzin's Hypothesis, resolvable space.

The author thanks Dr. W.W. Comfort for invaluable guidance in his Ph.D study, and the Mathematics Department of Wesleyan University for generous support.

(C)2003 American Mathematical Society 
The first question was asked privately by W.W. Comfort and S. Garcia-Ferreira. In [6], they have shown that every totally bounded topological group is strongly extraresolvable. In [14], the authors proved that every strongly extraresolvable space is $\omega$-resolvable and a countable space with certain properties is strongly extraresolvable. For instance, every countable weakly Fréchet-Urysohn space is extraresolvable. Here a space $X$ is weakly Fréchet-Urysohn if for every $x \in X$, whenever $x \in \bar{A}$ there is a countably infinite family $\mathcal{A}$ of pairwise disjoint finite subsets of $A$ such that for every neighborehood $V$ of $x,\{B \in \mathcal{A}: V \cap B=\emptyset\}$ is finite (see [14]). Question 0.1 then arises naturally.

The second question was asked first in [3] and then in [5]. In [9], F.W. Eckertson proved a consistent result in the positive direction: assuming the existence of a crowded, strong $P_{\kappa}$, HI space, it is possible to prove that there exists an $\omega$-resolvable Tychonoff space which is not maximally resolvable.

In this paper, we introduce the so-called KID-expansion. Using this tool, we can expand the topology of a Tychonoff space in such a way that a fixed family of pairwise disjoint dense subsets is still a family of dense subsets in the expansion, while any other family of pairwise disjoint dense subsets with cardinality big enough will not be a family of dense subsets in the expansion. We shall give a negative answer to the first question by proving that if a countable Tychonoff space $X$ has a family of infinitely many pairwise disjoint OHI dense subsets, then $X$ has a KIDexpansion that is $\omega$-resolvable but not strongly extraresolvable. For the second question, we prove that, under Luzin's Hypothesis $\left(2^{\omega}=2^{\omega_{1}}\right)$, there exists an $\omega$-resolvable Tychonoff space of size $\omega_{1}$ which is not maximally resolvable.

The concept of an independent family was introduced in [16]. See also [18], [8]. We use the definition in [18]. Let $X$ be a set and let $\mathcal{I}$ be a subfamily of $\mathcal{P}(X)$. The family $\mathcal{I}$ is called a uniformly independent family on $X$ if for any distinct $X_{1}, \ldots, X_{n}, Y_{1}, \ldots, Y_{m}$ in $\mathcal{I}$, the intersection $X_{1} \cap \ldots \cap X_{n} \cap\left(X \backslash Y_{1}\right) \cap \ldots \cap\left(X \backslash Y_{m}\right)$ has cardinality $|X|$.

For a space $\langle X, \mathcal{T}\rangle$, the density is denoted by $d(X)$ and the open density $o d(X)$ is defined as $\operatorname{od}(X)=\min \{d(U): U$ is an empty open set in $X\}$. When $\operatorname{od}(X)>\aleph_{0}$, we call $X$ an open-hereditarily inseparable space (OHIS). Given a space $X$, let $S(X)$ be the smallest cardinal number $\kappa$ such that there is no disjoint family of size $\kappa$ of non-empty open subsets of $X$ (see [8]). If $A$ is a subset of the space $\langle X, \mathcal{T}\rangle$, then we use $\langle A, \mathcal{T}\rangle$ to denote the set $A$ with the topology inherited from $\langle X, \mathcal{T}\rangle$.

For other notation and terminology, the reader is referred to [10].

\section{KID-EXPANSIONS}

Definition 1.1. A dense partition of a space $X$ is a partition of $X$ which consists of dense subsets.

We want to expand a topology in such a way that some selected dense subsets are added as new open sets, while a fixed dense partition in the original topology is preserved as a dense partition in the expansion.

Lemma 1.2. Let $\left\{X_{\alpha}: \alpha<\kappa\right\}$ be a dense partition of $\langle X, \mathcal{T}\rangle$, and let $D$ be a dense subset of $\langle X, \mathcal{T}\rangle$. Then the topology $\mathcal{T}^{\prime}$ generated by $\mathcal{T} \cup\{D\}$ remains crowded. The topology $\mathcal{T}^{\prime}$ keeps each $X_{\alpha}$ dense if and only if for each $\alpha$ the set $D \cap X_{\alpha}$ is dense in $X_{\alpha}$ with its original subspace topology. 
Proof. $(\Longleftarrow)$ For each non-empty open set $O \in \mathcal{T}$, we have $(O \cap D) \cap X_{\alpha}=$ $O \cap\left(D \cap X_{\alpha}\right) \neq \emptyset$. Thus each $X_{\alpha}$ is still dense in $\mathcal{T}^{\prime}$. Since $D$ is dense in $X$, the expansion $\mathcal{T}^{\prime}$ must be crowded.

$(\Longrightarrow)$ Suppose that for some $\alpha<\kappa$ the set $D \cap X_{\alpha}$ is not dense in $X_{\alpha}$. Then there exists some non-empty open set $O \in \mathcal{T}$ such that $O \subset X \backslash\left(D \cap X_{\alpha}\right)$. Since $(O \cap D)$ is open in $\mathcal{T}^{\prime}$ and $X_{\alpha}$ is dense in $\mathcal{T}^{\prime}$, we have $(O \cap D) \cap X_{\alpha} \neq \emptyset$. However $(O \cap D) \cap X_{\alpha}=O \cap\left(D \cap X_{\alpha}\right)=\emptyset$, which is a contradiction.

We start from a matrix of dense subsets, that is, we begin with a $\kappa \times \tau$ dense partition $\mathcal{D}=\left\{D_{\alpha}^{\gamma}: \alpha<\kappa, \gamma<\tau\right\}$ of $X$. When expanding, we want to keep the union of each row $\bigcup\left\{D_{\alpha}^{\gamma}: \alpha<\kappa\right\}$ dense. In order to get a crowded expansion, we use an independent family on each column.

When expanding, we are going to kill any dense partition with cardinality big enough by adding some new open sets which are members of that partition excluding some "good" subsets. The concept of "good" sets with respect to a partition is introduced in Definition 1.3 We shall show in the next section that, fixing a dense partition $\mathcal{D}$, every other dense partition $\mathcal{D}^{\prime}$ with cardinality big enough contains an element that is a good set with respect to $\mathcal{D}$.

Definition 1.3. Let $\langle X, \mathcal{T}\rangle$ be a space and let $\mathcal{E}=\left\{E_{\alpha}: \alpha<\kappa\right\}$ be an arbitrary partition of $\langle X, \mathcal{T}\rangle$. A subset $K \subseteq X$ is called a "good" set with respect to $\mathcal{E}$ if for every $\alpha<\kappa$ the set $K \cap E_{\alpha}$ has empty interior in the subspace $\left\langle E_{\alpha}, \mathcal{T}\right\rangle$. $X$.

Clearly, there are at most $2^{|X|}$-many good sets with respect to any partition of

It is a well-known result (see [18] Lemma 24.8) that for any infinite cardinal $\kappa$, there exists a uniformly independent family of size $2^{\kappa}$ on $\kappa$. Now we define the so-called KID-expansion.

Definition 1.4. Let $\langle X, \mathcal{T}\rangle$ be a Tychonoff space.

(1) Let $\kappa, \tau$ be two cardinals such that $\kappa \geq \omega$ and $\kappa \geq \tau \geq 1$.

(2) Let $\mathcal{D}=\left\{X_{\alpha}^{\gamma}: \alpha<\kappa, \gamma<\tau\right\}$ be a $\kappa \times \tau$-matrix of pairwise disjoint crowded subsets of $\langle X, \mathcal{T}\rangle$. Define $X^{\gamma}:=\bigcup_{\alpha<\kappa} X_{\alpha}^{\gamma}$, the union of the $\gamma$-th row.

(3) Let $\mathcal{I}=\left\{I_{\beta}: \beta<2^{\kappa}\right\}$ be a uniformly independent family on $\kappa$ of size $2^{\kappa}$.

(4) Let $\mathcal{K}=\left\{K_{\beta}: \beta<2^{\kappa}\right\}$ be a family of good sets with respect to $\mathcal{D}$ with possible repetitions.

(5) For each $\beta<2^{\kappa}$, define $W_{\beta}=\bigcup\left\{X_{\alpha}^{\gamma}: \alpha \in I_{\beta}, \gamma<\tau\right\} \backslash K_{\beta}$, and let $\mathcal{T}_{\beta}=\left\{W_{\beta}, X \backslash W_{\beta}\right\}$.

(6) Let $\mathcal{T}_{\mathcal{K I D}}$ denote the topology generated by $\mathcal{T}$ together with all $\mathcal{T}_{\beta}, \beta<2^{\kappa}$. Then $\mathcal{T}_{\mathcal{K I D}}$ is the KID-expansion of $\mathcal{T}$ via $\mathcal{K}, \mathcal{I}$, and $\mathcal{D}$.

Clearly, any KID-expansion is a Tychonoff expansion. In this paper, we shall only consider KID-expansions with $\mathcal{D}$ a $(\kappa \times \tau)$-matrix of pairwise disjoint dense subsets.

KID-expansions have some good properties.

Lemma 1.5. If $X_{\alpha}^{\gamma}$ is $O H I$ and dense in $X$ for all $\alpha<\kappa, \gamma<\tau$, then $\mathcal{T}_{\mathcal{K} \mathcal{D D}}$ has dispersion character $\geq \operatorname{od}(\mathcal{T}) \cdot \tau$, and each $X^{\gamma}=\bigcup_{\alpha<\kappa} X_{\alpha}^{\gamma}$ is a dense subset in $\mathcal{T}_{\mathcal{K} \mathcal{I D}}$.

Proof. For $\beta<2^{\kappa}$ and $C \in\left\{W_{\beta}, X \backslash W_{\beta}\right\}$, we define $I_{C}=I_{\beta}$ if $C=W_{\beta}$ and $I_{C}=\kappa \backslash I_{\beta}$ otherwise. 
For distinct $\beta_{0}, \ldots, \beta_{m-1}<2^{\kappa}$, and $C_{i} \in\left\{W_{\beta_{i}}, X \backslash W_{\beta_{i}}\right\}$, consider $I_{C_{0}} \cap \ldots \cap I_{C_{m-1}}$. Since $\mathcal{I}$ is an independent family, this set is not empty. Let $p \in I_{C_{0}} \cap \ldots \cap I_{C_{m-1}}$. Then for each $\gamma<\tau$ and $i<m$, we have $X_{p}^{\gamma} \backslash K_{\beta_{i}} \subset C_{i}$ if $C_{i}=W_{\beta_{i}}$ (equivalently, if $I_{C_{i}} \in \mathcal{I}$ ), and we have $X_{p}^{\gamma} \subseteq C_{i}$ otherwise (that is, if $\kappa \backslash I_{C_{i}} \in \mathcal{I}$ ).

We claim that for each $K \in \mathcal{K}$ and any $\alpha<\kappa, \gamma<\tau$, the set $X_{\alpha}^{\gamma} \cap K$ is nowhere dense in $\left\langle X_{\alpha}^{\gamma}, \mathcal{T}\right\rangle$. Otherwise, let $U$ be the interior of $\operatorname{cl}\left(X_{\alpha}^{\gamma} \cap K\right)$ in the space $\left\langle X_{\alpha}^{\gamma}, \mathcal{T}\right\rangle$. Then $X_{\alpha}^{\gamma} \cap K$ is dense in $U$. Since $X_{\alpha}^{\gamma} \cap K$ has an empty interior in $\left\langle X_{\alpha}^{\gamma}, \mathcal{T}\right\rangle$, for any nonempty open set $U^{\prime} \subseteq U$, we have $\left(X_{\alpha}^{\gamma} \backslash K\right) \cap U^{\prime} \neq \emptyset$. Hence $X_{\alpha}^{\gamma} \backslash K$ is also dense in $U$. But this is a contradiction, since $X_{\alpha}^{\gamma}$ is open-hereditarily irresolvable. The claim is proved.

Hence for each $\gamma<\tau$, the union $\bigcup_{i<m} X_{p}^{\gamma} \cap K_{\beta_{i}}$ is nowhere dense in $\left\langle X_{p}^{\gamma}, \mathcal{T}\right\rangle$. This is equivalent to the condition that for each $\gamma<\tau$ the set $\bigcap_{i<m} X_{p}^{\gamma} \backslash K_{\beta_{i}}$ is dense in $\left\langle X_{p}^{\gamma}, \mathcal{T}\right\rangle$.

Thus, for any $O \in \mathcal{T}$ the statement $O \cap C_{0} \cap \ldots \cap C_{m-1} \supseteq O \cap C_{0} \cap \ldots \cap C_{m-1} \cap X_{p}^{\gamma} \supseteq$ $\left(O \cap X_{p}^{\gamma}\right) \cap\left(X_{p}^{\gamma} \backslash K_{\beta_{0}}\right) \cap \ldots \cap\left(X_{p}^{\gamma} \backslash K_{\beta_{m-1}}\right)=\left(O \cap X_{p}^{\gamma}\right) \cap\left(\bigcap_{i<m} X_{p}^{\gamma} \backslash K_{\beta_{i}}\right) \neq \emptyset$ holds for all $\gamma<\tau$. Hence $O \cap C_{0} \cap \ldots \cap C_{m-1}$ cannot be a singleton. Therefore, $\mathcal{T}_{\mathcal{K I D}}$ has no isolated points and $\Delta\left(\mathcal{T}_{\mathcal{K I D}}\right) \geq \operatorname{od}(\mathcal{T}) \cdot \tau$. From the above proof, one can also see that each $X_{\gamma}, \gamma<\tau$, is dense in $\mathcal{T}_{\mathcal{K I D}}$.

The second useful property is given in the following theorem, which shows that some KID-expansions preserve the Souslin property.

Theorem 1.6. If each $X_{\alpha}^{\gamma}$ is $O H I$, and $\langle X, \mathcal{T}\rangle$ has the Souslin property, then $\left\langle X, \mathcal{T}_{\mathcal{K I D}}\right\rangle$ has the Souslin property, too.

Proof. It is enough to show that every uncountable family of basic open sets has a pair with non-empty intersection.

Let $\left\{U_{\beta}: \beta<\omega_{1}\right\}$ be a family of basic open sets, where each $U_{\beta}$ is of the form: $U_{\beta}=O_{\beta} \cap C_{\beta_{1}} \cap \ldots \cap C_{\beta_{k_{\beta}}}$ with $C_{\beta_{i}} \in\left\{W_{\beta_{i}}, X \backslash W_{\beta_{i}}\right\}, O_{\beta} \in \mathcal{T}, k_{\beta}<\omega$ and $\beta_{1}, \ldots, \beta_{k_{\beta}}<2^{\kappa}$.

Consider the family of all index sets $A_{\beta}=\left\{\beta_{1}, \ldots, \beta_{k_{\beta}}\right\}, \beta<\omega_{1}$. This is a family of finite subsets of $\omega_{1}$. According to the $\Delta$-lemma (see 18 Lemma 22.6), there exists a subset $A \subseteq \omega_{1}$ and a finite set $J$ such that $|A|=\omega_{1}$ and for distinct $\beta_{1}, \beta_{2} \in A$, we have $A_{\beta_{1}} \cap A_{\beta_{2}}=J$.

For each $\theta \in J$, either $C_{\theta}=W_{\theta}$ or $C_{\theta}=X \backslash W_{\theta}$. Since $J$ is finite, there exists an uncountable subset $B \subseteq A$ and a fixed choice $D_{\theta} \in\left\{W_{\theta}, X \backslash W_{\theta}\right\}$ of $C_{\theta}$ for each $\theta \in J$ such that for any $\beta \in B$, the set $U_{\beta}$ can be written as

$$
O_{\beta} \cap\left(\bigcap_{\theta \in J} D_{\theta}\right) \cap\left(\bigcap_{\theta \in\left\{\beta_{1}, \ldots, \beta_{k_{\beta}}\right\} \backslash J} C_{\theta}\right) .
$$

Then for any $\zeta, \beta \in B$, we have

$$
U_{\zeta} \cap U_{\beta}=\left(O_{\zeta} \cap O_{\beta}\right) \cap\left(\bigcap_{\theta \in J} D_{\theta}\right) \cap\left(\bigcap_{\theta \in\left\{\zeta_{1}, \ldots, \zeta_{k_{\zeta}}\right\} \backslash J} C_{\theta}\right) \cap\left(\bigcap_{\theta \in\left\{\beta_{1}, \ldots, \beta_{k_{\beta}}\right\} \backslash J} C_{\theta}\right) .
$$

For each $\beta \in B$ and each $i<k_{\beta}$, let $I_{C_{\beta_{i}}}=I_{\beta_{i}}$ if $C_{\beta_{i}}=W_{\beta_{i}}$ and $I_{C_{\beta_{i}}}=\kappa \backslash I_{\beta_{i}}$ otherwise. Since $\mathcal{I}$ is an independent family, for distinct $\zeta, \beta \in B$, we have

$$
\left(\bigcap_{\theta \in J} I_{C_{\theta}}\right) \cap\left(\bigcap_{\theta \in\left\{\zeta_{1}, \ldots, \zeta_{k_{\zeta}}\right\} \backslash J} I_{C_{\theta}}\right) \cap\left(\bigcap_{\theta \in\left\{\beta_{1}, \ldots, \beta_{k_{\beta}}\right\} \backslash J} I_{C_{\theta}}\right) \neq \emptyset .
$$


Let $p$ be a point in this set. Since each $X_{\alpha}^{\gamma}$ is OHI, for each $\gamma<\tau$ and some nowhere dense set $K$, the following holds:

$$
X_{p}^{\gamma} \backslash K \subseteq\left(\bigcap_{\theta \in J} D_{\theta}\right) \cap\left(\bigcap_{\theta \in\left\{\zeta_{1}, \ldots, \zeta_{k_{\zeta}}\right\} \backslash J} C_{\theta}\right) \cap\left(\bigcap_{\theta \in\left\{\beta_{1}, \ldots, \beta_{k_{\beta}}\right\} \backslash J} C_{\theta}\right) .
$$

Since $\langle X, \mathcal{T}\rangle$ has the Souslin property, there exist $\zeta \neq \beta \in B$ such that $O_{\zeta} \cap O_{\beta} \neq$ $\emptyset$. For this $\zeta$ and $\beta$, we have $U_{\zeta} \cap U_{\beta} \neq \emptyset$, since $U_{\zeta} \cap U_{\beta}$ contains the intersection of a non-empty open set $O_{\zeta} \cap O_{\beta}$ with the union $\bigcup\left\{X_{p}^{\gamma} \backslash K: \gamma<\tau\right\}$ of dense sets in $\langle X, \mathcal{T}\rangle$. Hence $\left\{U_{\alpha}: \alpha<\omega_{1}\right\}$ cannot be pairwise disjoint. Therefore, $\mathcal{T}_{\mathcal{K} \mathcal{I D}}$ has the Souslin property.

\section{Answering Question 0.1}

Definition 2.1. Let $\mathcal{D}=\left\{D_{\alpha}: \alpha<\kappa\right\}$ be a family of dense subsets. If for any $\alpha \neq \beta<\kappa$, the set $D_{\alpha} \cap D_{\beta}$ is finite, then we call $\mathcal{D}$ a family of dense subsets with finite intersections; in short, a $D F I$-family.

Remark 2.2. Suppose that $\mathcal{T}^{\prime} \supset \mathcal{T}$ are two topologies on $X$. Then any DFI-family in $\left\langle X, \mathcal{T}^{\prime}\right\rangle$ is also a DFI-family in $\langle X, \mathcal{T}\rangle$.

From the following lemma, we can see that given a dense partition $\mathcal{D}=\left\{D_{\alpha}\right.$ : $\alpha<\kappa\}$, any sufficiently large DFI-family contains an element that is good with respect to $\mathcal{D}$.

Lemma 2.3. Let $\kappa, \tau$ be two infinite cardinals such that $\tau\langle c f(\kappa)$. Let $\langle X, \mathcal{T}\rangle$ be a space, and let $\left\{X_{\alpha}: \alpha<\tau\right\}$ be a family of dense subsets such that $X=\bigcup_{\alpha<\tau} X_{\alpha}$ and $S\left(X_{\alpha}\right) \leq \kappa$ for all $\alpha<\tau$. Then for any DFI-family $\mathcal{E}$ of size $\kappa$ in $X$, all but $<\kappa$-many members $E$ of $\mathcal{E}$ satisfy the following: For any $\alpha<\tau$ the set $X_{\alpha} \cap E$ has empty interior in $\left\langle X_{\alpha}, \mathcal{T}\right\rangle$.

Proof. Suppose that there exists a subfamily $\mathcal{E}^{\prime} \subseteq \mathcal{E}$ of size $\kappa$ such that for each member $E \in \mathcal{E}^{\prime}$, there is $\sigma(E)<\tau$ such that $X_{\sigma(E)} \cap E$ has a non-empty interior in $X_{\sigma(E)}$. Under the map $\sigma: \mathcal{E}^{\prime} \rightarrow \tau$ given by $E \rightarrow \sigma(E)$, some $\alpha<\tau$ has preimage of cardinality $\kappa$, so we assume without loss of generality, passing to a suitable subfamily of $\mathcal{E}^{\prime}$ if necessary, that for some fixed $\alpha<\tau$ we have: for each $E \in \mathcal{E}^{\prime}$, the set $X_{\alpha} \cap E$ has non-empty interior in $\left\langle X_{\alpha}, \mathcal{T}\right\rangle$. Since $\mathcal{E}^{\prime}$ is a DFI-family, for distinct $E, E^{\prime} \in \mathcal{E}^{\prime}$, we have $i n t_{X_{\alpha}}\left(X_{\alpha} \cap E\right) \cap i n t_{X_{\alpha}}\left(X_{\alpha} \cap E^{\prime}\right) \subseteq i n t_{X_{\alpha}}\left(X_{\alpha} \cap E \cap E^{\prime}\right)=\emptyset$ in the space $\left\langle X_{\alpha}, \mathcal{T}\right\rangle$. Hence in $\left\langle X_{\alpha}, \mathcal{T}\right\rangle$, the family $\left\{\right.$ int $\left._{X_{\alpha}}\left(X_{\alpha} \cap E\right): E \in \mathcal{E}^{\prime}\right\}$ consists of $\kappa$-many pairwise disjoint non-empty open subsets of $X_{\alpha}$, contradicting the assumption that $S\left(X_{\alpha}\right) \leq \kappa$.

Corollary 2.4. Let everything be as in the above lemma. If each $X_{\alpha}$ is also OHI, then all but $<\kappa$-many members in $\mathcal{E}$ satisfy the following: For any $\alpha<\tau$ the set $X_{\alpha} \cap E$ is nowhere dense in $\left\langle X_{\alpha}, \mathcal{T}\right\rangle$.

Proof. Use the same argument together with the fact (from OHI) that if for some $E \in \mathcal{E}$ the set $E \cap X_{\alpha}$ has empty interior in $\left\langle X_{\alpha}, \mathcal{T}\right\rangle$, then $E \cap X_{\alpha}$ is nowhere dense in $\left\langle X_{\alpha}, \mathcal{T}\right\rangle$.

Now we prove that every countable Tychonoff space with a dense partition consisting of infinitely many $\mathrm{OHI}$ dense subsets has a KID-expansion that is $\omega$ resolvable but not strongly extraresolvable. 
Theorem 2.5. Suppose $\langle X, \mathcal{T}\rangle$ is a countable Tychonoff space with a dense partition $\mathcal{D}=\left\{X_{n}^{i}: n, i<\omega\right\}$ of OHI dense subsets. Let $\mathcal{K}=\left\{K_{\beta}: \beta<2^{\omega}\right\}$ enumerate the family of all good sets with respect to $\mathcal{D}$ (if there are less than $c$ many good sets, we repeat one of them $c$ times). For any uniformly independent family $\mathcal{I}=\left\{I_{\beta}: \beta<2^{\omega}\right\}$ on $\omega$, the KID-expansion $\mathcal{T}_{\mathcal{K} \mathcal{I D}}$ via $\mathcal{K}, \mathcal{I}$, $\mathcal{D}$, is an $\omega$-resolvable Tychonoff space that has no uncountable DFI-family. Hence it is not strongly extraresolvable.

Proof. By the results of Lemma 1.5 in the previous section, we know that $\mathcal{T}_{\mathcal{K I D}}$ is $\omega$-resolvable. We will show the rest.

Since for every $\beta<2^{\omega}$ we have $W_{\beta} \cap K_{\beta}=\emptyset$, the set $K_{\beta}$ is not dense in $\mathcal{T}_{\mathcal{K} \mathcal{I D}}$. If there exists an uncountable DFI-family in $X$, then by Corollary 2.4, it contains some element in $\mathcal{K}$. As we have just shown, this element is not dense in $\mathcal{T}_{\mathcal{K} \mathcal{I D}}$, which gives a contradiction. Hence $\mathcal{T}_{\mathcal{K} \mathcal{I D}}$ has no uncountable DFI-family.

Theorem 2.6. There exist countable Tychonoff spaces that are $\omega$-resolvable but not strongly extraresolvable.

Proof. It is proved in [1] Theorem 2.3 that the Cantor cube $\{0,1\}^{c}$ has a dense countable, irresolvable subspace $D$. By a standard argument, $D$ contains a nonempty HI open subset of the form $D \cap U$ for some basic open set $U$ in $\{0,1\}^{c}$. Since any basic open set of $\{0,1\}^{c}$ is homeomorphic to $\{0,1\}^{c}$ itself, we know that there exists a countable, HI, dense subspace $E$ in $\{0,1\}^{c}$.

Let $\langle E\rangle$ denote the subgroup of $\{0,1\}^{c}$ generated by $E$. Then the quotient $\{0,1\}^{c} /\langle E\rangle$ has size $2^{c}$ with each coset homeomorphic to $\langle E\rangle$ and dense in $\{0,1\}^{c}$. Let $X=\bigcup_{n} x_{n} E$, where $\left\{x_{n}: n<\omega\right\}$ is a countable infinite subset in $\{0,1\}^{c}$, so that the cosets $x_{n}\langle E\rangle$ are distinct. Then $X$ with the subspace topology from $\{0,1\}^{c}$ is a Tychonoff space with a dense partition of HI dense subsets and has the Souslin property. By Theorem 2.5 $X$ has a KID-expansion that is $\omega$-resolvable but not strongly extraresolvable.

\section{Answering Question 0.2}

In [3] and [5], the authors have asked whether $\omega$-resolvable, not maximally resolvable, Tychonoff spaces exist. In [9], Eckertson gave an example under the assumption of the existence of "a crowded, strong $P_{\kappa}$, HI space". In the same paper, it was shown that the existence of such a space is equiconsistent with the existence of a measurable cardinal. In this section, we show that, under Luzin's Hypothesis $\left(2^{\omega}=2^{\omega_{1}}\right)$, we can find an example of size $\omega_{1}$.

Let us first introduce the following concept.

Definition 3.1. A space $\langle X, \mathcal{T}\rangle$ is $\kappa$-condensed if there exists a family $\mathcal{K}$ of nowhere dense subsets such that $|\mathcal{K}|=\kappa$ and every nowhere dense subset of $X$ is contained in some element of $\mathcal{K}$.

For cardinals $\kappa, \tau$, we define as usual $\tau^{<\kappa}=\Sigma\left\{\tau^{\theta}: \theta<\kappa\right\}$.

Lemma 3.2. Let $\langle X, \mathcal{T}\rangle$ be a space. If $w(X) \leq \tau$, then $X$ is $\tau^{<S(X)}$-condensed.

Proof. Let $\mathcal{B}=\left\{B_{\alpha}: \alpha<\tau\right\}$ be a base for $\langle X, \mathcal{T}\rangle$. For any maximal pairwise disjoint subfamily $\mathcal{A}$ of $\mathcal{B}$, we have $|\mathcal{A}|<S(X)$ and $\bigcup \mathcal{A}$ is dense in $\langle X, \mathcal{T}\rangle$.

Let $\mathcal{K}^{\prime}$ be the family of all sets expressible as the union of a maximal pairwise disjoint subfamily of $\mathcal{B}$. An easy counting argument shows that $\left|\mathcal{K}^{\prime}\right| \leq \tau^{<S(X)}$. 
Clearly each element in $\mathcal{K}^{\prime}$ is dense and open in $\langle X, \mathcal{T}\rangle$. Let $\mathcal{K}=\left\{X \backslash K: K \in \mathcal{K}^{\prime}\right\}$. We show that every nowhere dense subset of $\langle X, \mathcal{T}\rangle$ is contained in some element in $\mathcal{K}$.

Let $K$ be a nowhere dense subset of $X$. Let $\mathcal{A}$ be a maximal pairwise disjoint subfamily of $\mathcal{B}$ such that each element in $\mathcal{A}$ is contained in $X \backslash K$. Then $\bigcup \mathcal{A} \subseteq X \backslash K$ and $\bigcup \mathcal{A} \in \mathcal{K}^{\prime}$. Hence $(X \backslash \bigcup \mathcal{A}) \in \mathcal{K}$ and $K \subseteq(X \backslash \bigcup \mathcal{A})$.

Now, we prove the following useful theorem.

Theorem 3.3. Let $\langle X, \mathcal{T}\rangle$ be a Tychonoff space, and let $\tau<\operatorname{cf}(S(\langle X, \mathcal{T}\rangle))$. Suppose $\langle X, \mathcal{T}\rangle$ is $2^{\tau}$-condensed, and has a dense partition consisting of $\tau$-many $O H I$ dense subsets. Then $\langle X, \mathcal{T}\rangle$ has a KID-expansion with dispersion character $\geq \operatorname{od}(\mathcal{T}) \cdot \tau$ that is $\tau$-resolvable but not $S(\langle X, \mathcal{T}\rangle)$-resolvable.

Proof. Let $\mathcal{D}=\left\{X_{\alpha}^{\gamma}: \alpha, \gamma<\tau\right\}$ be a dense partition of $\langle X, \mathcal{T}\rangle$ such that each $X_{\alpha}^{\gamma}$ is OHI. Since $\langle X, \mathcal{T}\rangle$ is $2^{\tau}$-condensed, and a nowhere dense subset in a dense subspace of $\langle X, \mathcal{T}\rangle$ is also nowhere dense in $\langle X, \mathcal{T}\rangle$, each dense subset of $\langle X, \mathcal{T}\rangle$ is also $2^{\tau}$-condensed. Hence there is a family $\mathcal{K}$ of good sets with respect to $\mathcal{D}$ such that $|\mathcal{K}|=2^{\tau}$ and every nowhere dense subset of $X$ is contained in some element in $\mathcal{K}$. Let us write $\mathcal{K}$ as $\left\{K_{\beta}: \beta<2^{\tau}\right\}$.

Now let $\mathcal{I}=\left\{I_{\beta}: \beta<2^{\tau}\right\}$ be a uniformly independent family on $\tau$. By Lemma 1.5. $\mathcal{T}_{\mathcal{K I D}}$ is $\tau$-resolvable, and it has dispersion character $\geq \operatorname{od}(\mathcal{T}) \cdot \tau$. We show that $\left\langle X, \mathcal{T}_{\mathcal{K} \mathcal{I D}}\right\rangle$ is not $S(\langle X, \mathcal{T}\rangle)$-resolvable.

We use the same argument as used in the proof of Theorem 2.5 to show that $\mathcal{I}_{\mathcal{K} \mathcal{I D}}$ has no dense partition of size $S(\langle X, \mathcal{T}\rangle)$. By Corollary 2.4. every dense partition of size $S(\langle X, \mathcal{T}\rangle)$ in $\langle X, \mathcal{T}\rangle$ contains some good set $K$ with respect to $\mathcal{D}$. By our definition of the family $\mathcal{K}$, this set $K$ is contained in some $K_{\beta}$ for some $\beta<2^{\tau}$. But in our definition of the KID-expansion, we have $W_{\beta} \cap K_{\beta}=\emptyset$, and hence $W_{\beta} \cap K=\emptyset$. Therefore, $K$ is not dense in $\mathcal{T}_{\mathcal{K} \mathcal{I D}}$ and $\mathcal{T}_{\mathcal{K} \mathcal{I D}}$ has no dense partition of size $S(\langle X, \mathcal{T}\rangle)$. Hence $\left\langle X, \mathcal{T}_{\mathcal{K} \mathcal{I D}}\right\rangle$ is not $S(\langle X, \mathcal{T}\rangle)$-resolvable.

Corollary 3.4. If $\langle X, \mathcal{T}\rangle$ satisfies the following conditions, then it has a KIDexpansion that is $\omega$-resolvable but not maximally resolvable.

(1) It is a Tychonoff space of size $\omega_{1}$ and has the Souslin property,

(2) it is an OHIS space and has a dense partition consisting of $\omega$-many $O H I$ subsets, and

(3) it is $2^{\omega}$-condensed.

Tychonoff spaces which satisfy the first two conditions in Corollary 3.4 exist in ZFC. To show this, it is enough to prove the following theorem.

Theorem 3.5. There exists an irresolvable dense subset $D$ of the Cantor cube $\{0,1\}^{2^{\omega_{1}}}$ such that $|D|=\operatorname{od}(D)=\omega_{1}$.

Proof. Let $K:=\{0,1\}^{2^{\omega_{1}}}$. Let $D_{1}$ be a dense subset of size $\omega_{1}$ in $K$. Consider the Cantor cube $\{0,1\}^{\omega_{1}}$. Fix a point $x \in\{0,1\}^{\omega_{1}}$. Then the $\sigma$-product $D_{2}:=\{y: y \in$ $\left.\{0,1\}^{\omega_{1}},\left|\left\{\alpha<\omega_{1}: y(\alpha) \neq x(\alpha)\right\}\right|<\omega\right\}$ is a dense set in $\{0,1\}^{\omega_{1}}$. In fact, we have $\left|D_{2}\right|=o d\left(D_{2}\right)=\omega_{1}$.

Let $D=D_{1} \times D_{2}$. Then $D$ is a dense subset in $K \times\{0,1\}^{\omega_{1}}=K$. Moreover, the set $D$ satisfies $|D|=o d(D)=\omega_{1}$.

Much as in the proof of Theorem 2.3 in [1], we now construct from the set $D$ an irresolvable dense subset in $K$ with size and open density $\omega_{1}$. 
List $D$ as $\left\{d_{\alpha}: \alpha<\omega_{1}\right\}$, and let $\left\{A_{\beta}: \beta<2^{\omega_{1}}\right\}$ enumerate the family of all infinite subsets of $\omega_{1}$. We define by induction for each $\beta<\tau, \tau<2^{\omega_{1}}$, a dense subset $D_{\beta}$ of the space $K \times\{0,1\}^{\beta}$ in the following way:

(1) the set $D_{\beta}:=\left\{d_{\alpha}^{\beta}: \alpha<\omega_{1}\right\}$ is dense in $K \times\{0,1\}^{\beta}$, and $\left|D_{\beta}\right|=\operatorname{od}\left(D_{\beta}\right)=$ $\omega_{1}$

(2) $d_{\alpha}^{\beta} \mid \gamma=d_{\alpha}^{\gamma}$ for all $\alpha<\omega_{1}$ and $0 \leq \gamma<\beta$;

(3) if both $H_{\beta}:=\left\{d_{\alpha}^{\beta}: \alpha \in A_{\beta}\right\}$ and $D_{\beta} \backslash H_{\beta}$ are dense in $D_{\beta}$, then the set $H_{\beta}^{\prime}:=\left\{d_{\alpha}^{\beta+1}: \alpha \in A_{\beta}\right\}$ is open in $D_{\beta+1}$.

Let $d_{\alpha}^{0}=d_{\alpha}$ for every $\alpha<\omega_{1}$. The set $D_{0}=\left\{d_{\alpha}^{0}: \alpha<\omega_{1}\right\}$ satisfies (1)-(3).

If $\beta$ is a limit ordinal, then let $d_{\alpha}^{\beta}=\bigcup\left\{d_{\alpha}^{\gamma}: \gamma<\beta\right\}$ for each $\alpha<\omega_{1}$. It is easy to see that $D_{\beta}=\left\{d_{\alpha}^{\beta}: \alpha<\omega_{1}\right\}$ satisfies (2) and (3). For (1), we only need to show that if each $\gamma<\beta$ satisfies $\operatorname{od}\left(D_{\gamma}\right)=\omega_{1}$, then $\operatorname{od}\left(D_{\beta}\right)=\omega_{1}$. Choose $(n+m)$-many ordinals $\gamma_{1}, \ldots, \gamma_{n}, \zeta_{1}, \ldots, \zeta_{m}<2^{\omega_{1}}+\beta$, and consider the corresponding open subset $U:=\left\{d_{\alpha}^{\beta}: d_{\alpha}^{\beta}\left(\gamma_{i}\right)=0, d_{\alpha}^{\beta}\left(\zeta_{j}\right)=1\right\}$ in $D_{\beta}$.

Let $\theta<2^{\omega_{1}}+\beta$ be an ordinal that is larger than all $\gamma_{i}$ 's and $\zeta_{j}$ 's. Since the projection map from $K \times\{0,1\}^{\beta}$ to $K \times\{0,1\}^{\theta}$ is open and continuous, it maps $U$ to an open subset $U^{\prime}$ in $D_{\theta}$, and also maps any dense subset of $U$ to some dense subset in $U^{\prime}$. Since $\operatorname{od}\left(D_{\theta}\right)=\omega_{1}$, we have $d(U)=\omega_{1}$. Therefore, it is true that $\operatorname{od}\left(D_{\beta}\right)=\omega_{1}$.

Let $\beta=\gamma+1$. If $D_{\gamma}$ is already irresolvable, then we stop the inductive construction. If both $H_{\gamma}=\left\{d_{\alpha}^{\gamma}: \alpha \in A_{\beta}\right\}$ and $D_{\gamma} \backslash H_{\gamma}$ are dense in $D_{\gamma}$, then set $\left.d_{\alpha}^{\beta}\right|_{\gamma}=d_{\alpha}^{\gamma}$ and $d_{\alpha}^{\beta}(\gamma)=1$ if $\alpha \in A_{\beta}$ and $d_{\alpha}^{\beta}(\gamma)=0$ otherwise. If $\left\{H_{\gamma}, D_{\gamma} \backslash H_{\gamma}\right\}$ is not a dense partition of $D_{\gamma}$, choose an arbitrary dense partition $\left\{Z, D_{\gamma} \backslash Z\right\}$ of $D_{\gamma}$. Set $d_{\alpha}^{\beta} \mid \gamma=d_{\alpha}^{\gamma}$ and $d_{\alpha}^{\beta}(\gamma)=1$ if $d_{\alpha}^{\beta} \in Z$ and $d_{\alpha}^{\beta}(\gamma)=0$ otherwise, and let $H_{\gamma}=\left\{\left.d_{\alpha}^{\beta}\right|_{\gamma}: d_{\alpha}^{\beta}(\gamma)=0\right\}$.

Again, we only need to check that $D_{\beta}$ satisfies $\operatorname{od}\left(D_{\beta}\right)=\omega_{1}$. Take $(n+m)$-many ordinals $\gamma_{1}, \ldots, \gamma_{n}, \zeta_{1}, \ldots, \zeta_{m}<2^{\omega_{1}}+\beta$, and consider the corresponding open subset $U:=\left\{d_{\alpha}^{\beta}: d_{\alpha}^{\beta}\left(\gamma_{i}\right)=0\right.$ and $\left.d_{\alpha}^{\beta}\left(\zeta_{i}\right)=1\right\}$ in $D_{\beta}$. If all $\gamma_{i}$ 's and $\zeta_{j}$ 's are less than $\beta$, then $d(U)=\omega_{1}$ by the same argument for the limit ordinal case. Otherwise, one of these $\gamma_{i}$ 's and $\zeta_{j}$ 's is equal to $\beta$, say $\gamma_{1}$. Let $U_{1}:=\left\{d_{\alpha}^{\gamma}: d_{\alpha}^{\gamma}\left(\gamma_{i}\right)=0\right.$ for all $i>1$ and $\left.d_{\alpha}^{\gamma}\left(\zeta_{i}\right)=1\right\}$. Then $U_{1}$ is an open subset in $D_{\gamma}$ and $U=\left\{d_{\alpha}^{\beta}: d_{\alpha}^{\beta} \mid \gamma \in H_{\gamma} \cap U_{1}\right\}=$ $\left\{d_{\alpha}^{\beta}: d_{\alpha}^{\beta} \mid \gamma \in U_{1}, d_{\alpha}^{\beta}(\gamma)=0\right\}$. Since $H_{\gamma}$ is dense in $D_{\gamma}$ and $\operatorname{od}\left(D_{\gamma}\right)=\omega_{1}$, we have $d\left(H_{\gamma} \cap U_{1}\right)=\omega_{1}$, which implies $d(U)=\omega_{1}$. Hence $\operatorname{od}\left(D_{\beta}\right)=\omega_{1}$.

Clearly the induction stops after $\tau$ steps for some ordinal $\tau \leq 2^{\omega_{1}}$. It is easy to see that the set $D_{\tau}=\left\{d_{\alpha}^{\tau}: \alpha<\omega_{1}\right\}$ is irresolvable in $K \times\{0,1\}^{\tau}$ and $\operatorname{od}\left(D_{\tau}\right)=$ $\left|D_{\tau}\right|=\omega_{1}$.

Remark 3.6. It is proved by a different method in [7] that for any infinite cardinal $\kappa$, there exists an irresolvable dense subset $D$ in $\{0,1\}^{2^{\kappa}}$ such that $|D|=\operatorname{od}(D)=\kappa$.

Theorem 3.7. Assume $2^{\omega}=2^{\omega_{1}}$. There exists a Tychonoff $\omega$-resolvable space of size $\omega_{1}$ which is not maximally resolvable.

Proof. By Theorem 3.5, there exists a dense irresolvable subset $D \subset\{0,1\}^{2^{\omega_{1}}}$ such that $|D|=\operatorname{od}(D)=\omega_{1}$. By a standard argument, we know that $D$ contains a nonempty open subset of the form $D \cap U$ for some basic open set $U$ in $\{0,1\}^{2^{\omega_{1}}}$ such that $D \cap U$ with the topology inherited from that of $\{0,1\}^{2^{\omega_{1}}}$ is hereditarily irresolvable. Certainly $\operatorname{od}(D \cap U)=\operatorname{od}(D)=\omega_{1}$. Since $U$ is homeomorphic to $\{0,1\}^{2^{\omega_{1}}}$, we can assume that $D$ can be chosen so that $D$ is HI and $|D|=\operatorname{od}(D)=\omega_{1}$. 
Consider the subgroup $\langle D\rangle$ generated by $D$. Let $X:=\bigcup_{n} x_{n} D$, where $\left\{x_{n}\right.$ : $n<\omega\}$ is a subset of $\{0,1\}^{\omega_{1}}$ of size $\omega$ such that the cosets $x_{n}\langle D\rangle$ are distinct. Then $X$ with the topology inherited from $\{0,1\}^{2^{\omega_{1}}}$ satisfies the first two conditions in Corollary [3.4] Hence $X$ satisfies all the three conditions in Corollary 3.4 when Luzin's Hypothesis is assumed. Therefore, the space $X$ has a KID-expansion that is $\omega$-resolvable but not maximally resolvable.

Since a space satisfying the three conditions in Corollary 3.4 can be embedded into $R^{c}$, where $R$ is the real line with the usual topology, a natural question is the following.

Question 3.8. Is there an OHIS, irresolvable, dense subset of the product $Q^{c}$ (equivalently, of $\left.\{0,1\}^{c}\right)$ ?

We show, in the following theorem, that a positive answer to this question will give a space which satisfies all three conditions in Corollary 3.4

Theorem 3.9. If there exists an OHIS, irresolvable, dense subset in the product $Q^{c}$, then there exists an $\omega$-resolvable Tychonoff space which is not maximally resolvable.

Proof. Let $D$ be an OHIS, irresolvable, dense subset in $Q^{c}$. Without loss of generality, using a similar argument as that in the proof of Theorem 3.7 if necessary, we assume that $D$ with the topology inherited from $Q^{c}$ is hereditarily irresolvable. Since $Q^{c}$ is dense in $R^{c}$, the set $D$ is also dense in $R^{c}$. Consider the quotient $R^{c} / Q^{c}$. Let $X:=\bigcup_{n} x_{n} D$, where the set $\left\{x_{n}: n<\omega\right\}$ is a countable infinite subset of $R^{c}$ such that the cosets $x_{n} Q^{c}$ are distinct. Then $X$ with the topology inherited from $R^{c}$ satisfies the first two conditions in Corollary 3.4. The space $X$ satisfies the third condition too, since $w(X)=w\left(Q^{c}\right)=w\left(R^{c}\right)=c$. Hence, by Corollary 3.4, the space $X$ has a KID-expansion which is $\omega$-resolvable, but not maximally resolvable.

\section{ACKNOWLEDGMENT}

The author is very grateful to the referee. Because of the referee's work, the exposition of this paper has been significantly improved from the original version. The author also thanks Dr. Comfort and the Editor for their efforts on this paper.

\section{REFERENCES}

[1] O.T. Alas, M. Sanchis, M.G. Tkacenko, V.V. Tkachuk, R.G. Wilson, Irresolvable and submaximal spaces: Homogeneity versus $\sigma$-discreteness and new ZFC examples, Top. Appl. 107 (2000), 259-273. MR 2002f:54004

[2] J.G. Ceder, On maximally resolvable spaces, Fund. Math. 55 (1964), 87-93. MR 29:582

[3] J.G. Ceder and T. Pearson, On products of resolvable spaces, Pacific J. Math. 22 (1967), 31-45. MR 36:841

[4] W.W. Comfort and Li Feng, The union of resolvable spaces is resolvable, Math. Japon. 38 (1993), 413-414. MR 94d:54084

[5] W.W. Comfort and S. Garcia-Ferreira, Resolvability: a selective survey and some new results, Top. Appl. 74 (1996), 149-167. MR 97i:54005

[6] W.W. Comfort and S. Garcia-Ferreira, Dense subsets of maximally almost periodic groups, Proc. Amer. Math. Soc. 129 (2001), 593-599. MR 2001e:22001

[7] W.W. Comfort and Wanjun Hu, Maximal independent families and a topological consequence, http://at.yorku.ca/i/d/e/c/27.htm, 2001.

[8] W.W. Comfort, S.A. Negrepontis, The Theory of Ultrafilters, Springer-Verlag, 1974. MR 53:135 
[9] F.W. Eckertson, Resolvable, not maximally resolvable spaces, Top. Appl. 79 (1997), 1-11. MR 98i:54019

[10] R. Engelking, General Topology, Heldermann, Berlin, 1989. MR 91c:54001

[11] E.K. van Douwen, Applications of maximal topologies, Top. Appl. 51 (1993), 125-139. MR 94h:54012

[12] A.G. El'kin, On the maximal resolvability of products of topological spaces, Soviet Math Dokl. 10 (1969), 659-662. MR 40:1977

[13] L. Feng, Strongly exactly n-resolvable spaces of arbitrarily large dispersion character, Top. Appl. 105 (2000), 31-36. MR 2001e:54059

[14] S. Garcia-Ferreira, V.I. Malykhin and A.H. Tomita, Extraresolvable spaces, Top. Appl. 101 (2000), 257-271. MR 2000k:54005

[15] L. Gillman and M. Jerison, Rings of Continuous Functions, Springer-Verlag, 1960. MR 53:11352

[16] F. Hausdorff, Über zwei Sätze von G. Fichtenholz und L. Kantorovitch, Studia Math. 6 (1936), 18-19.

[17] E. Hewitt, A problem of set-theoretic topology, Duke Math. J. 10 (1943), 309-333. MR 5:46e

[18] T. Jech, Set Theory, second edition, Springer-Verlag, 1997. MR 99b:03061

[19] I. Juhász, Cardinal Functions in Topology - Ten Years Later, second edition, Mathematical Centre Tracts 123, Amsterdam, 1980. MR 82a:54002

[20] V. I. Malykhin, Irresolvability is not descriptively good (preprint).

[21] K. Kunen, A. Szymanski and F. Tall, Baire resolvable spaces and ideal theory, Prace Nauk., Ann. Math. Sil. 2 (14)(1986), 98-107. MR 87j:54048

[22] K. Kunen and F. Tall, On the consistency of the non-existence of Baire irresolvable spaces, http://at.yorku.ca/v/a/a/a/27.htm, 1998.

[23] T.L. Pearson, Some sufficient conditions for maximal-resolvability, Canad. Math. Bull. 14 (1971), 191-196. MR 46:9920

Department of Mathematics, Wesleyan University, Middletown, Connecticut 06459 E-mail address: whu@claude.math.wesleyan.edu

Current address: Department of Mathematics and Computer Science, Albany State University, Albany, Georgia 31705

E-mail address: whu@asurams.edu 